International Mathematical Forum, Vol. 8, 2013, no. 14, 675 - 684 HIKARI Ltd, www.m-hikari.com

\title{
Fuzzy Strong Regular Congruence Triples for an $E$-inversive Semigroup ${ }^{\dagger}$
}

\author{
Yabing Shi, Zhenji Tian* and Tianjie Zhang \\ School of Sciences \\ Lanzhou University of Technology \\ Lanzhou, Gansu, 730050, P.R. China \\ syb475471891@163.com
}

Copyright (c) 2013 Yabing Shi et al. This is an open access article distributed under the Creative Commons Attribution License, which permits unrestricted use, distribution, and reproduction in any medium, provided the original work is properly cited.

\begin{abstract}
In this paper, we introduce the concept of fuzzy strong regular congruence triple of a fuzzy strong regular congruence on an $E$-inversive semigroup, and then we show that each fuzzy strong regular congruence on an $E$-inversive semigroup is uniquely determined by its fuzzy strong regular congruence triple. Finally, it is obtained that there exists a oneone correspondence between fuzzy strong regular congruence triples and fuzzy strong regular congruences on an $E$-inversive semigroup.
\end{abstract}

Keywords: Fuzzy strong regular congruence; Fuzzy strong regular congruence triple; $\mathcal{L}^{*}\left(\mathcal{R}^{*}\right)$-regular part; Green* equivalence relations; $E$-inversive semigroup

\section{Introduction and preliminaries}

The concepts of fuzzy sets and fuzzy congruences on inverse semigroups are introduced by Al-Thukair in [1] and he sets up a one-one correspondence between the set of fuzzy congruences and the set of fuzzy congruence pairs on an inverse

$\dagger$ Supported by the National Natural Science Foundation of China(Grant N. 11261030)

* Corresponding author: Zhenji Tian (E-mail: zjtian@lut.cn) 
semigroup.Wang [8] and Tian [6] studied the relationships between fuzzy congruences and fuzzy congruence triples on completely 0-simple semigroup and completely simple semigroup respectively. Francis Pastijn and Mario Petrich [2] studied the congruence triples by using Green's relations on regular semigroup and concluded that congruence relation is uniquely determined by its associated triple. $\mathrm{Li}$ [3] introduced the concepts of fuzzy congruence triples and fuzzy congruences on a regular semigroup and he obtained that there exists a one-one correspondence between the fuzzy congruence and fuzzy congruence triples on a regular semigroup. Luo [5] first generalized Green' relations on arbitrary semigroups and introduced the strong regular congruence pairs for an $E$-inversive semigroup. It is proved that each strong regular congruence $\rho$ on an $E$-inversive semigroup $S$ is uniquely determined by the strong regular congruence pair. In this paper, we introduce the concept of fuzzy strong regular congruence triple of a fuzzy strong regular congruence on an $E$-inversive semigroup, and then we show that each fuzzy strong regular congruence on an $E$-inversive semigroup is uniquely determined by its fuzzy strong regular congruence triple. Finally, it is obtained that there exists a one-one correspondence between fuzzy strong regular congruence triples and fuzzy strong regular congruences on an E-inversive semigroup.

\section{Definition and basic results}

An element $a$ of a semigroup $S$ is called regular if there exists $x$ such that $a x a=$ a. A semigroup $S$ is called regular semigroup if its all elements are regular. A semigroup $S$ is called $E$-inversive semigroup if there exists $x \in S$ such that $a x \in E(S)$. As usual, $E(S)$ is the set of all idempotents and $\operatorname{Reg}(S)$ is the set of regular elements of $S$. If $a$ is a regular element of $S, V(a)=\{x \in S \mid a x a=$ $a, x a x=x\}$ is the set of all inverses of $a$. An element $x \in S$ is called weak inverse of $a \in S$ if $x a x=x$. Devote by $W(a)$ the set of all weak inverses of $a \in S$.

Let $S$ be a semigroup and $e, f \in E(S)$, and let

$$
M(e, f)=\{g \in E(S) \mid g e=g=f g\}
$$

and

$$
S(e, f)=\{g \in E(S)) \mid g e=g=f g, e g f=e f\} .
$$

$S(e, f)$ is called the sandwith set of $e$ and $f$. Clearly, if $e, f \in E(S)$ and $e \mathcal{R} f(e \mathcal{L} f)$, then $S(e, f)=\{e\}(S(e, f)=\{f\})$. If $\rho$ is a congruence on $S$ and $h \in S(e, f)$, then $h \rho \in S(e \rho, f \rho)$. Note that in an E-inversive semigroup $S$, $M(e, f)$ is not empty for any $e, f \in E(S)$.

A congruence on an $E$-inversive semigroup $S$ is called to be strong regular if for any $a \in S$, there exists $a^{\prime} \in W(a)$ such that $a \rho a a^{\prime} a$. Clearly, in this case, $S / \rho$ is a regular semigroup. 
As the generalization of Green' relations on regular semigroup, Green* relations on $E$-inversive semigroup $S$ are defined by:

Definition 2.1 ${ }^{[5]}$ Let $S$ be a semigroup and $\tau$ be an equivalence relation on $E(S)$. Define the following binary relations on $S$ by, for $a, b \in S$,

$$
\begin{aligned}
a \mathcal{L}_{\tau}^{*} b \Leftrightarrow & \left(\forall a^{\prime} \in W(a)\right)\left(\exists b^{\prime} \in W(b)\right)\left(a^{\prime} a \tau b^{\prime} b\right) \\
& \&\left(\forall b^{\prime} \in W(b)\right)\left(\exists a^{\prime} \in W(a)\right)\left(a^{\prime} a \tau b^{\prime} b\right) ; \\
a \mathcal{R}_{\tau}^{*} b \Leftrightarrow & \left(\forall a^{\prime} \in W(a)\right)\left(\exists b^{\prime} \in W(b)\right)\left(a a^{\prime} \tau b b^{\prime}\right) \\
& \&\left(\forall b^{\prime} \in W(b)\right)\left(\exists a^{\prime} \in W(a)\right)\left(a a^{\prime} \tau b b^{\prime}\right) ; \\
a \mathcal{H}_{\tau} b \Leftrightarrow & \left\{\begin{array}{l}
\left(\forall a^{\prime} \in W(a)\right)\left(\exists b^{\prime} \in W(b)\right)\left(a a^{\prime} \tau b b^{\prime}, a^{\prime} a \tau b^{\prime} b\right) \\
\left(\forall b^{\prime} \in W(b)\right)\left(\exists a^{\prime} \in W(a)\right)\left(a a^{\prime} \tau b b^{\prime}, a^{\prime} a \tau b^{\prime} b\right) .
\end{array}\right.
\end{aligned}
$$

If $\tau$ is the identity relation on $E(S)$, we use the notation $\mathcal{L}^{*}, \mathcal{R}^{*}$ in place of $\mathcal{L}_{\tau}^{*}, \mathcal{R}_{\tau}^{*}$, respectively. It is clear that $\mathcal{L}_{\tau}^{*}, \mathcal{R}_{\tau}^{*}, \mathcal{L}_{\tau}^{*} \cap \mathcal{R}_{\tau}^{*}$ and $\mathcal{H}_{\tau}$ are equivalence relations on $S$ and $\mathcal{H}_{\tau} \subseteq \mathcal{L}_{\tau}^{*} \cap \mathcal{R}_{\tau}^{*}$. Recall from Theorem 2.3 [4] that if $a, b$ are regular elements of a semigroup $S$, then $a \mathcal{L}^{*} b\left(a \mathcal{R}^{*} b, a\left(\mathcal{L}^{*} \cap \mathcal{R}^{*}\right) b\right)$ if and only if $a \mathcal{L} b(a \mathcal{R} b, a \mathcal{H} b)$.

Let $\rho$ be a congruence on a semigroup $S$. The restriction of $\rho$ to $E(S)$ is called the trace of $\rho$ and is denoted by $\operatorname{tr} \rho$. The subset $\left\{a \in S \mid a \rho a^{2}\right\}$ of $S$ is called the kernel of $\rho$ and is denoted by $\operatorname{ker} \rho$. From lemma 1.1 of [6], if $\rho$ is a strong regular congruence on an $E$-inversive semigroup $S$, then

$$
\operatorname{ker} \rho=\{a \in S \mid \exists e \in E(S), a \rho e\} .
$$

If $\gamma$ is an equivalence on a semigroup $S$, the greatest congruence on $S$ contained in $\gamma$ is denoted by $\gamma^{0}$. If $\Gamma$ is a family of equivalences on $S$, then $\cap_{\gamma \in \Gamma} \gamma^{0}=\left(\cap_{\gamma \in \Gamma} \gamma\right)^{0}(\operatorname{see}[2])$.

Lemma 2.2 ${ }^{[5]}$ Let $\rho$ be a strong regular congruence on an $E$-inversive semigroup $S$ with $\tau=\operatorname{tr} \rho$. Then

(1) $(e \rho) \mathcal{R}^{*}(f \rho) \Leftrightarrow e\left(\tau \mathcal{R}^{*} \tau\right) f \Leftrightarrow e \mathcal{R}_{\tau}^{*} f(e, f \in E(S))$;

(2) $(a \rho) \mathcal{R}^{*}(b \rho) \Leftrightarrow a \mathcal{R}_{\tau}^{*} b$;

(3) $\mathcal{R}_{\tau}^{*}=\rho \mathcal{R}^{*} \rho=\rho \vee \mathcal{R}^{*}$;

(4) $\left.\mathcal{R}_{\tau}^{*}\right|_{E(S)}=\tau \mathcal{R}^{*} \tau=\left.\left(\rho \vee \mathcal{R}^{*}\right)\right|_{E(S)}=\tau \vee\left(\left.\mathcal{R}^{*}\right|_{E(S)}\right)$;

(5) $\tau=\operatorname{tr}\left(\mathcal{L}_{\tau}^{*} \cap \mathcal{R}_{\tau}^{*}\right)^{0}=\tau \mathcal{L}^{*} \tau \cap \tau \mathcal{L}^{*} \tau$.

By lemma 2.7 in [2] and lemma 2.2, we can easily obtain the following lemma.

Lemma 2.3 Let $\rho$ be a strong regular congruence on an $E$-inversive semigroup $S$ with $\tau=\operatorname{tr} \rho$. Then

(1) $e \tau \mathcal{R}^{*} \tau f$

(2) $\varnothing \neq(e \tau)(f \tau) \cap E(S) \subseteq f \tau, \varnothing \neq(f \tau)(e \tau) \cap E(S) \subseteq e \tau$;

(3) $S(e, f) \subseteq e \tau, S(f, e) \subseteq f \tau$,

for any $e, f \in E(S)$. 
Lemma 2.4 Let $\Gamma$ be a family of strong regular congruences on an $E$-inversive semigroup $S$. Then

$$
\left.\left(\left(\cap_{\rho \in \Gamma} \rho\right) \vee \mathcal{R}^{*}\right)\right|_{E(S)}=\left.\left(\cap_{\rho \in \Gamma}\left(\rho \vee \mathcal{R}^{*}\right)\right)\right|_{E(S)}
$$

Proof. Let $e, f \in E(S)$ be such that $e\left(\cap_{\rho \in \Gamma}\left(\rho \vee \mathcal{R}^{*}\right)\right) f$. Then for every strong regular congruence $\rho \in \Gamma$, we have $e\left(\rho \vee \mathcal{R}^{*}\right) f$. Thus, if $g \in S(e, f)$ we have from lemma 2.2 and lemma 2.3 that $g \rho e$ for all $\rho \in \Gamma$. Further, $(g f) \rho=(g \rho)(f \rho)=(e \rho)(f \rho)=f \rho$ for all $\rho \in \Gamma$ since $(e \rho) \mathcal{R}^{*}(f \rho)$ by lemma $2.2(1),(3)$. Consequently

$$
e\left(\cap_{\rho \in \Gamma} \rho\right) g \mathcal{R}^{*} g f\left(\cap_{\rho \in \Gamma} \rho\right) f
$$

that is,

$$
e\left(\left(\cap_{\rho \in \Gamma} \rho\right) \vee \mathcal{R}^{*}\right) f
$$

Hence

$$
\left.\left.\left(\left(\cap_{\rho \in \Gamma} \rho\right) \vee \mathcal{R}^{*}\right)\right|_{E(S)} \supseteq\left(\cap_{\rho \in \Gamma}\left(\rho \vee \mathcal{R}^{*}\right)\right)\right|_{E(S)}
$$

holds and the reverse inclusion obviously holds.

Lemma 2.5 ${ }^{[5]}$ For any strong regular congruence $\rho$ on an $E$-inversive semigroup $S, K=\operatorname{ker} \rho, \tau=\operatorname{tr} \rho . a, b \in S$, we have

$$
a \rho b \Leftrightarrow a\left(\mathcal{L}_{\tau}^{*} \cap \mathcal{R}_{\tau}^{*}\right) b, a b^{\prime} \in K \text { for all } b^{\prime} \in W(b) .
$$

Let $X$ be a non-empty, a map $A: X \rightarrow[0,1]$ is called a fuzzy subset of $X$. Devote the set of all fuzzy subsets on $X$ by $F(X)$. Let $A \in F(X), t \in[0,1]$, define $A^{t}=\{a \in X \mid A(a) \geq t\}$, we call $A^{t}$ a $t$-cut set. Let $t \in[0,1)$, define $A^{\ell}=\{a \in X \mid A(a)>t\}$, we call $A^{\ell}$ a power $t$-cut set. The mapping $\rho$ : $X \times X \rightarrow[0,1]$ is called a fuzzy relation on $X$, let $\rho$ and $\sigma$ be fuzzy relations on $X, \rho \leq \sigma$ means that $\rho(a, b) \leq \sigma(a, b)$ for all $a, b \in X$. Their composition denoted by $\rho \circ \sigma$ and defined as:

$$
(\rho \circ \sigma)(a, c)=\sup \{\min \{\rho(a, b), \sigma(b, c)\}, b \in X\},
$$

for any $a, c \in X$. We denote $\rho \circ \sigma$ by $\rho \sigma$ for the sake of simplicity.

Let $t \in[0,1]$, define

$$
\rho^{t}=\{(a, b) \in X \times X \mid \rho(a, b) \geq t\} .
$$

Let $t \in[0,1)$, define

$$
\rho^{\ell}=\{(a, b) \in X \times X \mid \rho(a, b)>t\} .
$$

Let $\Gamma \subseteq F(X)$, define $\cap_{A \in \Gamma} A$ as

$$
\left(\cap_{A \in \Gamma} A\right)(x)=\inf \{A(x) \mid A \in \Gamma\}
$$


for all $x \in X$.

A fuzzy relation $\rho$ is called a fuzzy equivalence relation on a semigroup $S$ if

(1) $\rho(a, a)=1$ for all $a \in S$;

(2) $\rho(a, b)=\rho(b, a)$ for all $a, b \in S$;

(3) $\rho(a, c) \geq \min \{\rho(a, b), \rho(b, c)\}$ for all $a, b, c \in S$.

A fuzzy equivalence relation $\rho$ on a semigroup $S$ is called a fuzzy congruence if $\rho(a b, c d) \geq \min \{\rho(a, c), \rho(b, d)\}$ or $(\rho(a c, b c) \geq \rho(a, b)$ and $\rho(c a, c b) \geq \rho(a, b))$ for all $a, b, c, d \in S$.

Let $S$ be an $E$-inversive semigroup. A fuzzy congruence $\rho$ is called a fuzzy strong regular congruence on $S$ if there exists $a^{\prime} \in W(a)$ such that $\rho\left(a, a a^{\prime} a\right)=1$ for any $a \in S$. Denote by $F E(S)$ the set of all fuzzy equivalences of a semigroup $S, F C(S)$ the set of all fuzzy congruences and $F R C(S)$ the set of all fuzzy strong regular congruences. If $\rho \in F R C(S)$, then $K_{\rho}(a)=$ $\sup \{\rho(a, e) \mid e \in E(S)\}$ is called the fuzzy kernel of $\rho$ for all $a \in S$. If $\Gamma \subseteq F E(S)(\Gamma \subseteq F C(S))$, then $\vee_{\rho \in \Gamma} \rho$ is the least fuzzy equivalence(fuzzy congruence) relation containing all $\rho \in \Gamma, \cap_{\rho \in \Gamma} \rho$ is the greatest fuzzy equivalence(fuzzy congruence) relation contained in each $\rho \in \Gamma$. A fuzzy relation $A$ is called regular normal on $S$ if there exists $\rho \in F R C(S)$ such that $A=K_{\rho}$. For $A \in F R C(S)$, we define $\xi_{A}$ as:

$$
\xi_{A}(a, b)=\sup \left\{\rho(a, b) \mid K_{\rho}=A, \rho \in F R C(S)\right\} .
$$

It is clear that $\xi_{A}$ is the greatest fuzzy strong regular congruence in $\{\rho \in$ $\left.F R C(S) \mid K_{\rho}=A\right\}$.

Lemma 2.6 ${ }^{[3]}$ Let $\rho$ be a fuzzy relation on a semigroup $S$, then $\rho$ is a fuzzy equivalence relation if and only if $\rho^{\ell}$ is an equivalence relation on $S$ for all $t \in[0,1)$.

Lemma $2.7^{[3]}$ Let $\mu$ be a fuzzy equivalence relation on a semigroup $S . \mu^{0}$ is defined as $\mu^{0}(a, b)=\inf \left\{\mu(x a y, x b y) \mid x, y \in S^{1}\right\}$, if $\varnothing \neq \Gamma \subseteq F E(S)$, then

(1) $\mu^{0}$ is the greatest fuzzy congruence contained in $\mu$;

(2) $\cap_{\mu \in \Gamma} \mu^{0}=\left(\cap_{\mu \in \Gamma} \mu\right)^{0}$.

Let $S$ be a semigroup and $\tau$ be an equivalence relation on $E(S)$. Define the following fuzzy binary relations on $S$ by, for $a, b \in S$,

$$
\begin{gathered}
\mathcal{L}_{f}^{*}(a, b)= \begin{cases}1, & (a, b) \in \mathcal{L}_{\tau}^{*} . \\
0, & (a, b) \notin \mathcal{L}_{\tau}^{*} .\end{cases} \\
\mathcal{R}_{f}^{*}(a, b)= \begin{cases}1, & (a, b) \in \mathcal{R}_{\tau}^{*} . \\
0, & (a, b) \notin \mathcal{R}_{\tau}^{*} .\end{cases}
\end{gathered}
$$

It is clear that both of $\mathcal{L}_{f}^{*}$ and $\mathcal{R}_{f}^{*}$ are fuzzy equivalences. 


\section{$3 \quad$ Fuzzy strong regular congruence triples}

Let $\rho \in F E(S)$. Define fuzzy equivalence $\rho_{a}$ as $\rho_{a}(x)=\rho(a, x)$.

Lemma 3.1 ${ }^{[3]}$ Let $S$ be a semigroup.

(1) If $\rho, \sigma \in F E(S)$ and $\nu_{0}=\rho, \nu_{1}=\rho \sigma, \nu_{2}=\rho \sigma \rho, \nu_{3}=\rho \sigma \rho \sigma, \cdots$, then

$$
\rho \vee \sigma=\sup _{n=0,1,2, \cdots, \infty} \nu_{n}
$$

(2) If $\rho \in F E(S)(F C(S))$, then $\rho_{a}=\rho_{b}$ if and only if $\rho(a, b)=1$ for $a, b \in S$.

Let $S$ be an $E$-inversive semigroup. Let $\rho, \sigma \in F E(S)$ be such that $\rho \leq \sigma$. The fuzzy relation on $S / \rho=\left\{\rho_{a} \mid a \in S\right\}$, devote by $\sigma / \rho$, is defined as for all $a, b \in S,(\sigma / \rho)\left(\rho_{a}, \rho_{b}\right)=\sigma(a, b)$. where $\rho_{a}, \rho_{b} \in S / \rho$.

Lemma 3.2 Let $S$ be an $E$-inversive semigroup. Let $\rho, \sigma \in F E(S)$ be such that $\rho \leq \sigma$. Then $\sigma / \rho$ is a fuzzy equivalence relation on $S / \rho$.

Proof. Let $\rho, \sigma \in E F(S)$. Suppose that $(a, b)$ and $(c, d)$ in $S \times S$ such that $\sigma(a, b) \neq \sigma(c, d)$, then $\left(\rho_{a}, \rho_{b}\right) \neq\left(\rho_{c}, \rho_{d}\right)$. Otherwise, we have $\rho_{a}=\rho_{c}$ and $\rho_{b}=\rho_{d}$, which leads to $\rho(a, c)=1$ and $\rho(b, d)=1$. Since $\rho \leq \sigma$, we have $\rho(a, c) \leq \sigma(a, c)=1$ and $\rho(b, d) \leq \sigma(b, d)=1$. That is $\sigma(a, b)=\sigma(c, d)$, which contradicts the assumption. Thus, $\sigma / \rho$ is a mapping. It is a routine matter to show that $\sigma / \rho$ is a a fuzzy equivalence relation on $S / \rho$, that is, for all $\rho_{a}, \rho_{b}, \rho_{c} \in S / \rho$, it satisfies the following conditions:

(1) $(\sigma / \rho)\left(\rho_{a}, \rho_{a}\right)=\sigma(a, a)=1$;

(2) $(\sigma / \rho)\left(\rho_{a}, \rho_{b}\right)=\sigma(a, b)=\sigma(b, a)=(\sigma / \rho)\left(\rho_{b}, \rho_{a}\right)$;

(3) $(\sigma / \rho)\left(\rho_{a}, \rho_{c}\right)=\sigma(a, c) \geq \min \{\sigma(a, b), \sigma(b, c)\}$

$$
=\min \left\{(\sigma / \rho)\left(\rho_{a}, \rho_{b}\right),(\sigma / \rho)\left(\rho_{b}, \rho_{c}\right)\right\} \text {. }
$$

Let $S$ be an $E$-inversive semigroup and let $\alpha \in F E\left(S / \mathcal{L}_{f}^{*}\right), \beta \in F E\left(S / \mathcal{R}_{f}^{*}\right)$. Define $\bar{\alpha}, \bar{\beta}$ as follow:

$$
\bar{\alpha}(a, b)=\alpha\left(\left(\mathcal{L}_{f}^{*}\right)_{a},\left(\mathcal{L}_{f}^{*}\right)_{b}\right), \bar{\beta}(a, b)=\beta\left(\left(\mathcal{R}_{f}^{*}\right)_{a},\left(\mathcal{R}_{f}^{*}\right)_{b}\right)
$$

for all $a, b \in S$. It is easy to verify that $\bar{\alpha}, \bar{\beta} \in F E(S), \bar{\alpha}^{0}, \bar{\beta}^{0} \in F R C(S)$, and $\mathcal{L}_{f}^{*} \leq \bar{\alpha}, \mathcal{R}_{f}^{*} \leq \bar{\beta}$. A fuzzy equivalence relation $\alpha \in F E\left(S / \mathcal{L}_{f}^{*}\right)(\beta \in$ $\left.F E\left(S / \mathcal{R}_{f}^{*}\right)\right)$ is called regular normal, if $\alpha=\left(\bar{\alpha}^{0} \vee \mathcal{L}_{f}^{*}\right) / \mathcal{L}_{f}^{*}\left(\beta=\left(\bar{\beta}^{0} \vee \mathcal{R}_{f}^{*}\right) / \mathcal{R}_{f}^{*}\right)$. Let $\rho$ be a strong regular congruence on $S$. $\left(\rho \vee \mathcal{L}_{f}^{*}\right) / \mathcal{L}_{f}^{*}$ is called an $\mathcal{L}^{*}$-regular part of $\rho,\left(\rho \vee \mathcal{R}_{f}^{*}\right) / \mathcal{R}_{f}^{*}$ is called a $\mathcal{R}^{*}$-regular part of $\rho$.

Lemma 3.3 Let $S$ be an $E$-inversive semigroup and $\beta \in F E\left(S / \mathcal{R}_{f}^{*}\right)$. Then $\beta$ is regular normal if and only if $\beta$ is $\mathcal{R}^{*}$-regular part of some fuzzy regular congruences on $S$. 
Proof. If $\beta$ is regular normal, choose $\bar{\beta}^{0}$ in $F R C(S)$ such that $\beta$ is $\mathcal{R}^{*}$-regular part of $\bar{\beta}^{0}$.

Conversely, let $\beta$ be a $\mathcal{R}^{*}$-regular part of $\rho \in F R C(S)$, that is $\beta=(\rho \vee$ $\left.\mathcal{R}_{f}^{*}\right) / \mathcal{R}_{f}^{*}$. By lemma 3.2, we have $\bar{\beta}=\rho \vee \mathcal{R}_{f}^{*}$. Clearly, $\rho \leq \bar{\beta}^{0}$, thus, $\rho \vee \mathcal{R}_{f}^{*} \leq$ $\bar{\beta}^{0} \vee \mathcal{R}_{f}^{*}$. On the other hand, from lemma 3.1 know that $\bar{\beta}^{0} \leq \rho \vee \mathcal{R}_{f}^{*}$. Further, $\bar{\beta}^{0} \vee \mathcal{R}_{f}^{*} \leq \rho \vee \mathcal{R}_{f}^{*}$. Therefore, $\beta=\left(\rho \vee \mathcal{R}_{f}^{*}\right) / \mathcal{R}_{f}^{*}=\left(\bar{\beta}^{0} \vee \mathcal{R}_{f}^{*}\right) / \mathcal{R}_{f}^{*}$, and so $\beta$ is regular normal.

Lemma 3.4 Let $S$ be an $E$-inversive semigroup. If $\rho \in F R C(S)$, then $\rho \vee \mathcal{L}_{f}^{*}=$ $\rho \mathcal{L}_{f}^{*} \rho, \rho \vee \mathcal{R}_{f}^{*}=\rho \mathcal{R}_{f}^{*} \rho$.

Proof. It is easy to verify that $\rho \mathcal{L}_{f}^{*} \rho \leq \rho \vee \mathcal{L}_{f}^{*}$ and $\rho \mathcal{L}_{f}^{*} \rho$ is a fuzzy equivalence relation. Since $\rho \vee \mathcal{L}_{f}^{*}$ is the least fuzzy equivalence relation containing $\rho$ and $\mathcal{L}_{f}^{*}$, thus, $\rho \mathcal{L}_{f}^{*} \rho \geq \rho \vee \mathcal{L}_{f}^{*}$, that is $\rho \vee \mathcal{L}_{f}^{*}=\rho \mathcal{L}_{f}^{*} \rho$. Similarly, $\rho \vee \mathcal{R}_{f}^{*}=\rho \mathcal{R}_{f}^{*} \rho$.

By lemma 3.1 and lemma 2.4, we can easily obtain the following lemma.

Lemma 3.5 Let $S$ be an $E$-inversive semigroup.

(1) If $\mathcal{R}_{f}^{*}(a, c)=1, \mathcal{R}_{f}^{*}(b, d)=1$ and $\rho \in F R C(S)$, then $\left(\rho \vee \mathcal{R}_{f}^{*}\right)(a, b)=$ $\left(\rho \vee \mathcal{R}_{f}^{*}\right)(c, d)$ for any $a, b, c, d \in S$;

(2) If $\varnothing \neq \Gamma \subseteq F R C(S)$, then $\left(\left(\cap_{\rho \in \Gamma} \rho\right) \vee \mathcal{R}_{f}^{*}\right)(e, f)=\left(\cap_{\rho \in \Gamma}\left(\rho \vee \mathcal{R}_{f}^{*}\right)\right)(e, f)$ for any $e, f \in E(S)$.

Lemma 3.6 Let $S$ be an $E$-inversive semigroup. Then the mapping

$$
\theta: \rho \rightarrow \rho \vee \mathcal{R}_{f}^{*}(\rho \in F R C(S))
$$

is a complete lattice homomorphism $F R C(S)$ into $F E(S)$.

Proof. Let $\Gamma \subseteq F R C(S)$ and $a, b \in S$. It clear that $\left(\vee_{\rho \in \Gamma} \rho\right) \vee \mathcal{R}_{f}^{*} \geq \vee_{\rho \in \Gamma}(\rho \vee$ $\left.\mathcal{R}_{f}^{*}\right)$.

On the other hand, since $\vee_{\rho \in \Gamma} \rho \leq \vee_{\rho \in \Gamma}\left(\rho \vee \mathcal{R}_{f}^{*}\right)$, we have

$$
\left(\vee_{\rho \in \Gamma} \rho\right) \vee \mathcal{R}_{f}^{*} \leq\left(\vee_{\rho \in \Gamma}\left(\rho \vee \mathcal{R}_{f}^{*}\right)\right) \vee \mathcal{R}_{f}^{*}=\vee_{\rho \in \Gamma}\left(\rho \vee \mathcal{R}_{f}^{*}\right)
$$

Therefore

$$
\left(\vee_{\rho \in \Gamma} \rho\right) \vee \mathcal{R}_{f}^{*}=\vee_{\rho \in \Gamma}\left(\rho \vee \mathcal{R}_{f}^{*}\right)
$$

It follows that properties of Green* relations that there exist $e, f \in E(S)$ such that $e \mathcal{R}^{*} a$, and $f \mathcal{R}^{*} b$, by lemma 3.5, we have

$$
\begin{aligned}
\left(\cap_{\rho \in \Gamma}\left(\rho \vee \mathcal{R}_{f}^{*}\right)\right)(a, b) & =\left(\cap_{\rho \in \Gamma}\left(\rho \vee \mathcal{R}_{f}^{*}\right)\right)(e, f) \\
& =\left(\left(\cap_{\rho \in \Gamma} \rho\right) \vee \mathcal{R}_{f}^{*}\right)(e, f) \\
& =\left(\left(\cap_{\rho \in \Gamma} \rho\right) \vee \mathcal{R}_{f}^{*}\right)(a, b),
\end{aligned}
$$

that is

$$
\left(\cap_{\rho \in \Gamma} \rho\right) \vee \mathcal{R}_{f}^{*}=\bigcap_{\rho \in \Gamma}\left(\rho \vee \mathcal{R}_{f}^{*}\right)
$$


We have proved that the mapping $\theta: \rho \rightarrow \rho \vee \mathcal{R}_{f}^{*}$ is a complete lattice homomorphism.

Definition 3.7 A triple $(\alpha, A, \beta)$ consisting of regular normal fuzzy equivalence $\alpha$ in $F E\left(S / \mathcal{L}_{f}^{*}\right)$ and $\beta$ in $F E\left(S / \mathcal{R}_{f}^{*}\right)$ and a regular normal fuzzy subset $A$ on $S$, is a fuzzy strong regular congruence triple if

(1) $\bar{\alpha}=(\bar{\alpha} \cap \bar{\beta})^{0} \vee \mathcal{L}_{f}^{*}, \bar{\beta}=(\bar{\alpha} \cap \bar{\beta})^{0} \vee \mathcal{R}_{f}^{*}$;

(2) $A \leq K_{\alpha^{0}}, \bar{\alpha} \leq \xi_{A} \vee \mathcal{L}_{f}^{*}$

(3) $A \leq K_{\beta^{0}}, \bar{\beta} \leq \xi_{A} \vee \mathcal{R}_{f}^{*}$.

If this is the case, we define

$$
\rho_{(\alpha, A, \beta)}=\xi_{A} \cap(\bar{\alpha} \cap \bar{\beta})^{0} .
$$

Lemma 3.8 Let $S$ be an $E$-inversive semigroup. If $\rho \in F R C(S)$, then

$$
\rho=\xi_{K_{\rho}} \cap\left(\rho \vee \mathcal{L}_{f}^{*}\right)^{0} \cap\left(\rho \vee \mathcal{R}_{f}^{*}\right)^{0}
$$

Proof. It is clear that

$$
\begin{aligned}
\rho(a, b) & \leq\left(\xi_{K_{\rho}} \cap\left(\rho \vee \mathcal{L}_{f}^{*}\right)^{0} \cap\left(\rho \vee \mathcal{R}_{f}^{*}\right)^{0}\right)(a, b) \\
& =\min \left\{\xi_{K_{\rho}}(a, b),\left(\rho \vee \mathcal{L}_{f}^{*}\right)^{0}(a, b),\left(\rho \vee \mathcal{R}_{f}^{*}\right)^{0}(a, b)\right\}
\end{aligned}
$$

for any $a, b \in S, \rho \in F R C(S)$.

On the other hand, let $r=\min \left\{\left(\rho \vee \mathcal{L}_{f}^{*}\right)^{0}(a, b),\left(\rho \vee \mathcal{R}_{f}^{*}\right)^{0}(a, b), \xi_{K_{\rho}}(a, b)\right\}$. If $r=0$, then $\rho(a, b)=0$. Suppose that $r>0$, from lemma 3.4, for any $\varepsilon>0(\varepsilon<$ $r)$, if $t=r-\varepsilon$, then there exist $g, h \in S \operatorname{such}$ that $\min \left\{\rho(a, g), \mathcal{R}_{f}^{*}(g, h), \rho(h, b)\right\}>$ $t$, thus, $\left(a \rho^{\ell}\right) \mathcal{R}_{\tau}^{*}\left(b \rho^{\ell}\right)$. Similarly, we have $\left(a \rho^{\ell}\right) \mathcal{L}_{\tau}^{*}\left(b \rho^{\ell}\right)$. Therefore, $\left(a \rho^{\ell}\right)\left(\mathcal{L}_{\tau}^{*} \cap\right.$ $\left.\mathcal{R}_{\tau}^{*}\right)\left(b \rho^{\ell}\right)$. Since $K_{\rho}\left(a b^{\prime}\right) \geq \xi_{K_{\rho}}\left(a b^{\prime}, b b^{\prime}\right) \geq \xi_{K_{\rho}}(a, b)>t$, where $b^{\prime} \in W(b)$, then there exists $e=b b^{\prime} \in E(S)$ such that $\rho\left(a b^{\prime}, e\right)>t$. Thus, $a b^{\prime} \in k e r \rho^{\ell}$. It follows from lemma 2.5 that $\rho(a, b)>t=r-\varepsilon$. We obtain $\rho(a, b) \geq r$, by making use of arbitrariness of $\varepsilon$. Thus, $\rho(a, b)=r$. It means

$$
\rho=\xi_{K_{\rho}} \cap\left(\rho \vee \mathcal{L}_{f}^{*}\right)^{0} \cap\left(\rho \vee \mathcal{R}_{f}^{*}\right)^{0}
$$

Theorem 3.9 Let $S$ be an $E$-inversive semigroup. If $(\alpha, A, \beta)$ is a fuzzy strong regular congruence triple, then $\rho_{(\alpha, A, \beta)}$ is a unique fuzzy strong regular congruence $\rho$ on $S$ such that $\alpha$ is the $\mathcal{L}^{*}$-regular part of $\rho, A=K_{\rho_{(\alpha, A, \beta)}}$ and $\beta$ is the $\mathcal{R}^{*}$-regular part of $\rho$.

Conversely, if $\rho$ is a fuzzy strong regular congruence on $S$, then $(\alpha, A, \beta)=$ $\left(\left(\rho \vee \mathcal{L}_{f}^{*}\right) / \mathcal{L}_{f}^{*}, K_{\rho},\left(\rho \vee \mathcal{R}_{f}^{*}\right) / \mathcal{R}_{f}^{*}\right)$ is a fuzzy strong regular congruence triple for $S$ and $\rho=\rho_{(\alpha, A, \beta)}$. 
Proof. Let $(\alpha, A, \beta)$ be a fuzzy strong regular congruence triple. Then

$$
\begin{aligned}
\rho_{(\alpha, A, \beta)}\left(a, a a^{\prime} a\right) & =\left(\xi_{A} \cap(\bar{\alpha} \cap \bar{\beta})^{0}\right)\left(a, a a^{\prime} a\right) \\
& =\left(\xi_{A} \cap \bar{\alpha}^{0} \cap \bar{\beta}^{0}\right)\left(a, a a^{\prime} a\right) \\
& =\inf \left\{\xi_{A}\left(a, a a^{\prime} a\right), \bar{\alpha}^{0}\left(a, a a^{\prime} a\right), \bar{\beta}^{0}\left(a, a a^{\prime} a\right)\right\} \\
& =1
\end{aligned}
$$

for any $a \in S, a^{\prime} \in W(a)$. This shows that $\rho_{(\alpha, A, \beta)}$ is a fuzzy strong regular congruence.

By lemma 2.7 and definition 3.7, for all $x \in S$, we have

$$
\begin{aligned}
K_{\rho_{(\alpha, A, \beta)}}(x) & =K_{\left(\xi_{A} \cap(\bar{\alpha} \cap \bar{\beta})^{0}\right)}(x) \\
& =\sup \left\{\left(\xi_{A} \cap \bar{\alpha}^{0} \cap \bar{\beta}^{0}\right)(x, e) \mid e \in E(S)\right\} \\
& =K_{\xi_{A}}(x) \cap K_{\bar{\alpha}^{0}}(x) \cap K_{\bar{\beta}^{0}}(x) \\
& =A(x) \cap K_{\bar{\alpha}^{0}}(x) \cap K_{\bar{\beta}^{0}}(x) \\
& =A(x) .
\end{aligned}
$$

Further

$$
\begin{aligned}
\rho_{(\alpha, A, \beta)} \vee \mathcal{R}_{f}^{*} & =\left(\xi_{A} \cap(\bar{\alpha} \cap \bar{\beta})^{0}\right) \vee \mathcal{R}_{f}^{*} \\
& =\left(\xi_{A} \vee \mathcal{R}_{f}^{*}\right) \cap\left((\bar{\alpha} \cap \bar{\beta})^{0} \vee \mathcal{R}_{f}^{*}\right) \\
& =\left(\xi_{A} \vee \mathcal{R}_{f}^{*}\right) \cap \bar{\beta} \\
& =\bar{\beta}
\end{aligned}
$$

and thus

$$
\left(\rho_{(\alpha, A, \beta)} \vee \mathcal{R}_{f}^{*}\right) / \mathcal{R}_{f}^{*}=\beta
$$

is the $\mathcal{R}^{*}$-regular part of $\rho_{(\alpha, A, \beta)}$. Dually, $\alpha$ is the $\mathcal{L}^{*}$-regular part of $\rho_{(\alpha, A, \beta)}$.

Let $\rho \in F R C(S)$ be such that $\alpha$ is the $\mathcal{L}^{*}$-regular part of $\rho, A=K_{\rho}$ and $\beta$ is the $\mathcal{R}^{*}$-regular part of $\rho$. Then $K_{\rho}=A=K_{\rho_{(\alpha, A, \beta)}}$. By lemma 3.8, we have

$$
\begin{aligned}
\rho & =\xi_{K_{\rho}} \cap\left(\rho \vee \mathcal{L}_{f}^{*}\right)^{0} \cap\left(\rho \vee \mathcal{R}_{f}^{*}\right)^{0} \\
& =\xi_{A} \cap \bar{\alpha}^{0} \cap \bar{\beta}^{0} \\
& =\xi_{A} \cap(\bar{\alpha} \cap \bar{\beta})^{0} \\
& =\rho_{(\alpha, A, \beta)} .
\end{aligned}
$$

Conversely, let $\rho$ in $F R C(S)$ and let $\alpha=\left(\rho \vee \mathcal{L}_{f}^{*}\right) / \mathcal{L}_{f}^{*}, A=K_{\rho}$ and $\beta=$ $\left(\rho \vee \mathcal{R}_{f}^{*}\right) / \mathcal{R}_{f}^{*}$. By lemma 3.3 and its dual $\alpha$ and $\beta$ are regular normal fuzzy equivalences on $F E\left(S / \mathcal{L}_{f}^{*}\right)$ and $F E\left(S / \mathcal{R}_{f}^{*}\right)$, respectively. Thus, $A$ is a regular normal fuzzy subset on $S$.

We note that $\bar{\alpha}=\rho \vee \mathcal{L}_{f}^{*}$ and $\bar{\beta}=\rho \vee \mathcal{R}_{f}^{*}$. Since $\mathcal{R}_{f}^{*} \leq \bar{\beta}$, it follows that $(\bar{\alpha} \cap \bar{\beta})^{0} \vee \mathcal{R}_{f}^{*} \leq \bar{\beta}$. Further, since $\rho \leq(\bar{\alpha} \cap \bar{\beta})^{0}$, we have $\bar{\beta}=\rho \vee \mathcal{R}_{f}^{*} \leq$ $(\bar{\alpha} \cap \bar{\beta})^{0} \vee \mathcal{R}_{f}^{*}$. Therefore $\bar{\beta}=(\bar{\alpha} \cap \bar{\beta})^{0} \vee \mathcal{R}_{f}^{*}$ and Dually, $\bar{\alpha}=(\bar{\alpha} \cap \bar{\beta})^{0} \vee \mathcal{L}_{f}^{*}$. 
$K_{\rho}=A$ gives $\rho \leq \xi_{A}$ whence $\bar{\beta}=\rho \vee \mathcal{R}_{f}^{*} \leq \xi_{A} \vee \mathcal{R}_{f}^{*}$ and dually, $\bar{\alpha}=\rho \vee \mathcal{L}_{f}^{*} \leq$ $\xi_{A} \vee \mathcal{L}_{f}^{*}$. We have proved that $(\alpha, A, \beta)$ is a fuzzy strong regular congruence triple. From the first part of the proof we may now conclude that $\rho=\rho_{(\alpha, A, \beta)}$.

By lemma 3.6, we can easily obtain the following corollary.

Corollary 3.10 Let $F R C T(S)$ be the poset of all fuzzy strong regular congruence triples for an $E$-inversive semigroup $S$ under the partial order given by

$$
(\alpha, A, \beta) \leq\left(\alpha^{\prime}, A^{\prime}, \beta^{\prime}\right) \Leftrightarrow \alpha \leq \alpha^{\prime}, A \leq A^{\prime}, \beta \leq \beta^{\prime}
$$

Then the mappings

$$
\rho \rightarrow\left(\left(\rho \vee \mathcal{L}_{f}^{*}\right) / \mathcal{L}_{f}^{*}, K_{\rho},\left(\rho \vee \mathcal{R}_{f}^{*}\right) / \mathcal{R}_{f}^{*}\right),(\alpha, A, \beta) \rightarrow \rho_{(\alpha, A, \beta)}
$$

are mutually inverse isomorphisms of $F R C(S)$ and $F R C T(S)$.

\section{References}

[1] A.Fawzi, Al-Thukair, Fuzzy Congruence Pairs of Inverse Semigroup, Fuzzy Sets and Systems, 56 (1993), 117-122.

[2] F.Pastijn, M.Petrich, Congruences on Regular Semigroups. Trans AmerMath. Soc, 295 (1986), 607-633.

[3] Li Yonghua, Xu Chengxian, Fuzzy Congruence Triples for a Regular Semigroup. Journal of xi'an jiaotong university, 36 (2002), 418-421.

[4] Luo Yanfeng, Li Xiaoling, The Maximum Idempotent Separating Congruence on Eventually Regular Semigroups. Semigroup Forum, 74(2) (2007), 306-318.

[5] Luo Yanfeng, Fan Xingkui, Li Xiaoling, Regular Congruences on E-inversive Semigroup. Semigroup Forum, 76 (2008), 107-123.

[6] Ma Yunfeng, Tian Zhenji, Fuzzy Congruence on Completely Simple Semigroups. International Mathematical Forum, 64 (2011), 3199-3204.

[7] V.Murali, Fuzzy Equaivalence Relations, Fuzzy Sets and Systems, 87 (1989), 155-163.

[8] Wang Xijian, Fuzzy Congruence on Completely 0-simple Semigroups. Fuzzy Systems and Mathematics, 21(2) (2007), 65-71.

Received: January 20, 2013 